\title{
Uso não prescrito de metilfenidato por estudantes de uma universidade brasileira: fatores associados, conhecimentos, motivações e percepções
}

\author{
Non-prescribed use of methylphenidate by students of a Brazilian \\ university: associated factors, knowledge, motivations and \\ perceptions
}

\author{
Laís de Aquino Rodrigues ${ }^{1}$ (D), Nájila Aélida OliveiraViana² (D), Vinícius Silva Belo ${ }^{3}$ (D), \\ Carlos Alberto Pegolo da Gama ${ }^{3}$ (D), Denise Alves Guimarães ${ }^{3}$ (D) \\ 'Universidade Federal de São João Del Rei (UFSJ) - Heliodora (MG), Brasil. \\ ${ }^{2}$ Hospital Alberto Cavalcanti, Universidade Federal de São João Del Rei (UFSJ) - Divinópolis (MG), Brasil. \\ ${ }^{3}$ Universidade Federal de São João Del Rei (UFSJ), Campus Centro-Oeste - Divinópolis (MG), Brasil.
}

Como citar: Rodrigues LA, Viana NAO, Belo VS, Gama CAP, Guimarães DA. Uso não prescrito de metilfenidato por estudantes de uma universidade brasileira: fatores associados, conhecimentos, motivações e percepções. Cad Saúde Colet, 2021;29(4):463-473. https://doi.org/10.1590/1414-462X202129040437

\begin{abstract}
Resumo
Introdução: O metilfenidato, utilizado especialmente para tratar o Transtorno do Déficit de Atenção e Hiperatividade tem conquistado universitários que buscam melhorar o desempenho cognitivo. Objetivo: Investigar conhecimentos, motivações, percepções e perfil de uso não prescrito desse medicamento por estudantes de uma Universidade Federal de Minas Gerais. Método: Aplicação de questionário estruturado a 696 estudantes de Bioquímica, Enfermagem, Farmácia e Medicina, e análise descritiva e dos fatores associados ao uso, por meio de modelos de regressão logística. Resultados: Dos participantes, 96,7\% afirmaram conhecer o medicamento e desses, 4,3\% o utilizam/utilizavam por motivos não prescritos. 0 principal motivo de uso era o melhoramento cognitivo, e a universidade foi o principal local de início de uso. A maior parte dos usuários percebeu aumento na concentração e 50\% relatou ocorrência de efeitos indesejados. Houve maior chance de uso não prescrito entre homens, maiores de 21 anos, envolvidos em atividades extracurriculares e menor chance de uso entre estudantes de Enfermagem. Conclusão: $\mathrm{A}$ análise de diferentes aspectos relacionados com o uso não prescrito do metilfenidato propicia um melhor entendimento das situações vivenciadas entre universitários e aponta os riscos à saúde relacionados à busca por melhores desempenhos acadêmicos e estratégias para lidar com sobrecarga de atividades.
\end{abstract}

Palavras-chave: metilfenidato; universidade; cognição; motivação.

\begin{abstract}
Background: Methylphenidate, especially used to treat Attention Deficit Hyperactivity Disorder, has conquered over university students who seek to improve cognitive performance. Objective: To investigate knowledge, motivations, perceptions, and profile of non-prescribed use of this drug by students of a Federal University of Minas Gerais. Method: A structured questionnaire was applied to 696 students of Biochemistry, Nursing, Pharmacy, and Medicine, and descriptive analysis and associated factors, using
\end{abstract}

Trabalho realizado na Universidade Federal de São João Del Rei (UFSJ), Campus Centro-Oeste Dona Lindu, Divinópolis (MG), Brasil.

Correspondência: Laís de Aquino Rodrigues. E-mail: lais.aquinorodrigues@gmail.com

Fonte de financiamento: CNPq - Conselho Nacional de Desenvolvimento Científico e Tecnológico e FAPEMIG - Fundação de Amparo à Pesquisa do Estado de Minas Gerais.

Conflito de interesses: nada a declarar.

Recebido em: Out. 02, 2019. Aprovado em: Set. 06, 2020
Este é um artigo publicado em acesso aberto (Open Access) sob a licença Creative Commons distribuição e reprodução em qualquer meio, sem restrições desde que o trabalho original seja corretamente citado. 
logistic regression models. Results: Of the participants, $96.7 \%$ said they knew the medicine and of these, $4.3 \%$ use it/used it for reasons not prescribed. The main reason for using it was the cognitive improvement and the university was the main place of use. Most users noticed an increased concentration and $50 \%$ reported the occurrence of unwanted effects. There was a greater chance of non-prescribed use among men, over 21 years old, involved in extracurricular activities, and less chance of use among nursing students. Conclusion: Analysis of different aspects related to the non-prescribed use of Methylphenidate provides a better understanding of the situations experienced by university students and points to health risks related to the search for better academic performance and strategies for dealing with activity overload.

Keywords: methylphenidate; university; cognition; motivation.

\section{INTRODUÇÃO}

O metilfenidato ou cloridrato de metilfenidato, mais conhecido no Brasil pelos nomes comerciais Ritalina ${ }^{\circledast}$ (Novartis) e Concerta ${ }^{\circledast}$ (Janssen-Cilag), é a droga psicoativa prescrita mais consumida no mundo, com fins médicos e não médicos ${ }^{1-4}$. No Brasil, a prescrição de metilfenidato é autorizada legalmente apenas para tratar crianças com diagnóstico de Transtorno do Déficit de Atenção e Hiperatividade (TDAH), além de adultos com narcolepsia ou cataplexia, conforme indicações e faixas etárias aprovadas pela Agência Nacional de Vigilância Sanitária (ANVISA) ${ }^{1,3-7}$. O uso terapêutico do medicamento fora de tais situações não tem sua segurança e eficácia reconhecidas pelo órgão regulador ${ }^{6,7}$. Em crianças e adolescentes diagnosticados com TDAH, a literatura aponta que o medicamento, prescrito de forma adequada a partir de um diagnóstico correto, é eficaz para melhora de alguns sintomas como a desatenção, a dificuldade de manter o foco nas atividades, a atividade motora aumentada e a impulsividade ${ }^{6}$. Por esse motivo, é conhecido no Brasil como "a droga da obediência"4,6. Em pacientes que sofrem de distúrbios do sono como a narcolepsia, essa substância também melhora a capacidade de permanecer acordado ${ }^{8}$.

A descoberta de tais efeitos fez com que indivíduos saudáveis de diversas regiões do mundo começassem a usar o metilfenidato com o intuito de melhorar a atenção, a concentração e a memória, objetivando o chamado aprimoramento cognitivo9. Porém, devido ao alto risco de dependência química pelo uso de metilfenidato, esse medicamento foi incluído na categoria A3 (substâncias psicotrópicas) e, no Brasil, sua venda e distribuição são controladas. Seu uso pode também acarretar efeitos colaterais e intoxicações ${ }^{10}$. A eficácia do metilfenidato em relação à modalidade de uso não prescrito ou não médico é questionável, já que os estudos realizados não identificaram evidências consistentes de que o medicamento desempenhe

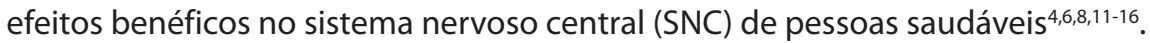

O consumo global de metilfenidato tem aumentado, o que contribui para fomentar o debate sobre a adequação médica na identificação de casos de TDAH e sobre o uso indiscriminado (não prescrito) por indivíduos que buscam nesse fármaco seus possíveis efeitos estimulantes ${ }^{2,17}$. Segundo estudo realizado em 2014, o consumo no Brasil passou de $94 \mathrm{~kg}$ em 2003 para 875 kg em 2012. No mesmo período, a produção nacional e a importação do medicamento cresceram $373 \%{ }^{1}$. Isso aponta para a necessidade de se estudar e compreender melhor o perfil de uso do metilfenidato, a fim de informar profissionais de saúde para que saibam indicá-lo de forma adequada, baseando-se em evidências científicas, além de fornecer subsídio para orientações à população ${ }^{1,5}$.

Estudos nacionais e internacionais vêm mostrando a tendência de uso não prescrito do metilfenidato principalmente no meio universitário, objetivando melhorar a performance acadêmica ${ }^{8,10,12,14,17,18}$. Um estudo nacional ${ }^{18}$ sobre as representações sociais de universitários relativas ao uso de metilfenidato mostrou que a pressão social para melhorar o desempenho é a principal motivação para uso não prescrito desse medicamento. Nesse contexto constatase o crescente consumo de psicofármacos como estratégia de potencialização cognitiva, de superação de limites e de alcance da perfeição, tornando as relações humanas e os conflitos pessoais dos indivíduos dependentes de medicamentos ${ }^{19,20}$.

Por outro lado, não há pesquisas que tenham avaliado conjuntamente as indicações do metilfenidato, as motivações para o uso da substância, as percepções sobre o uso e os efeitos 
indesejados percebidos em universitários. Além disso, nos estudos publicados sobre os fatores associados ao uso não prescrito do medicamento não foram utilizados modelos multivariados para a análise, o que pode fazer com que os resultados obtidos estejam sujeitos a situações de confusão. Assim, o presente estudo investiga conhecimentos, motivações, percepções e o perfil de uso não prescrito de metilfenidato por estudantes de uma Universidade Federal.

\section{MÉTODO}

Trata-se de um estudo epidemiológico seccional realizado no período de outubro de 2015 a março de 2016, com estudantes dos cursos de graduação em Bioquímica, Farmácia, Enfermagem e Medicina do campus Centro-Oeste da Universidade Federal de São João Del-Rei (UFSJ/CCO). Foram considerados como potenciais participantes da pesquisa todos os alunos regularmente matriculados nos quatro cursos mencionados da instituição de ensino, que perfazia um total de 1.303. Foram incluídos todos os estudantes que se dispuseram a responder voluntariamente um questionário autoaplicável, e que assinaram o Termo de Consentimento Livre e Esclarecido (TCLE).

Para a operacionalização da coleta de dados, os responsáveis pela realização da pesquisa fizeram contato com professores e representantes de cada uma das turmas dos diferentes cursos para apresentação da proposta e agendamento de datas e horários adequados para que fosse feito o encontro em cada turma. Nas datas previamente agendadas, os pesquisadores apresentaram a proposta da pesquisa para as turmas, formalizaram o convite, esclareceram dúvidas e deram prosseguimento à aplicação dos questionários e formalização da participação para aqueles que aceitaram participar da pesquisa. Foram aplicados 721 questionários e, desses, oito foram excluídos por conterem erro no preenchimento de questões. Assim, foram considerados 713 questionários válidos e, posteriormente, foram excluídos 17 questionários nos quais os participantes haviam declarado fazer uso do metilfenidato por indicação médica, uma vez que esta situação estava fora dos objetivos fixados para este estudo. Foram considerados nesta análise um total de 696 questionários que compuseram a amostra do presente estudo, representando $53,4 \%$ da população-alvo do estudo.

\section{Instrumentos}

Para a realização do estudo foi construído um questionário estruturado baseado em instrumentos utilizados previamente ${ }^{21-23}$, composto por 51 questões organizadas em quatro blocos: 1) informações sociodemográficas:idade; sexo; curso/período; graduações anteriores; situação conjugal; renda familiar; escolaridade dos responsáveis financeiros; etnia; religião; se o participante estava trabalhando no momento da pesquisa e carga horária de trabalho; participação em atividades extracurriculares e carga horária dessas atividades; 2 ) informações sobre o conhecimento do metilfenidato: conhecimentos sobre o nome comercial do medicamento e sua indicação; abordagem do medicamento e sua indicação na Universidade; influência das informações recebidas para que o participante cogitasse a possibilidade de utilização não médica; conhecimento sobre pessoas que fazem uso não médico para melhoria do desempenho acadêmico; se o participante avalia que a universidade propicia o uso de substâncias psicoativas; 3 ) informações sobre o uso e motivações para o uso do metilfenidato: uso prévio e uso atual do medicamento; tratamento de TDAH; motivo do uso não médico do medicamento; quando usou pela primeira vez; motivos de uso durante a vida escolar/ acadêmica; motivos para interrupção do uso; frequência de uso; forma de administração; dose; meio de obtenção; 4) percepção sobre o uso do metilfenidato: efeitos do uso não médico do medicamento; objetivos do uso e alcance desses objetivos; mudança na concentração e memória; avaliação de pontos positivos e negativos do uso; percepção de efeitos indesejáveis.

No questionário havia uma questão específica sobre uso anterior ou atual do metilfenidato (Ritalina ${ }^{\oplus}$ ou Concerta ${ }^{\circledR}$ ) para tratamento de Transtorno do Déficit de Atenção e Hiperatividade (TDAH). 


\section{Análise estatística}

Os dados obtidos foram tabulados no programa Epi info versão 7.2.0.1 e analisados por meio do programa $R$, versão 3.5.1. Foram analisadas inicialmente as variáveis (sexo, idade, religião, renda, curso de graduação, trabalho e realização de atividades extracurriculares) que poderiam atuar como fatores explicativos para uma maior ou menor chance de uso não prescrito do metilfenidato. Devido à dispersão das respostas entre os grupos da variável "etnia" e pela colinearidade da variável "renda" com a variável "escolaridade", as duas primeiras não foram analisadas. As variáveis relacionadas ao conhecimento, por sua vez, não foram consideradas como fatores potencialmente associados ao uso, pois poderiam ser, na verdade, resultantes de um maior interesse prévio pela substância ou mesmo um resultado do próprio uso. Sendo assim, para essas variáveis não seria possível estabelecer adequadamente a rede de causalidade com o desfecho.

Após a condução de análises descritivas, foi utilizado o teste de Qui-quadrado de Pearson e, para o ajuste das variáveis com valores de $\mathrm{P}<0,20$ na análise bivariada, foram construídos modelos multivariados de regressão logística. Após a execução de procedimentos não automáticos de seleção retroativa de variáveis, o modelo final foi composto por aquelas com valores de $\mathrm{P}<0,05$. Nessa etapa, as associações foram expressas por meio de Odds Ratio ajustados e de seus respectivos intervalos de $95 \%$ de confiança. Desse modo, o modelo de regressão logística foi utilizado para o controle da situação de confusão entre as variáveis modeladas. O resultado apresentado no modelo final demonstrou a força de cada uma das associações independentes entre as variáveis de exposição e o uso do metilfenidato. $O$ ajuste dos modelos foi avaliado e classificado adequado pelo teste de Hosmer e Lemeshow e pelos gráficos de:"resíduos padronizados", "normal Q-Q plot", "leverage" e "distância de Cook".

Foram ainda descritas as frequências absolutas e relativas das variáveis relacionadas ao conhecimento, às percepções, aos efeitos indesejados e ao perfil de uso entre os participantes que fizeram uso não prescrito do metilfenidato.

A pesquisa seguiu todas as recomendações éticas definidas pela Resolução 466/2012, tendo sido aprovada pelo Comitê de Ética em Pesquisa sob parecer no 1.137.594 de 13/07/2015, CAAE: 44803615.2.0000.5545. Todos os estudantes que aceitaram participar da pesquisa assinaram o Termo de Consentimento Livre e Esclarecido (TCLE).

\section{RESULTADOS}

Dos 696 estudantes, 495 (71,6\%) eram do sexo feminino, $196(28,4 \%)$ do sexo masculino e $5(7,1 \%)$ não responderam a essa questão. As faixas etárias mais prevalentes foram de 21 a 23 anos (37,9\%) e de 18 a 20 anos (36,3\%). O curso com maior participação no estudo foi o de Medicina (31,9\% dos participantes) seguido pelos cursos de Enfermagem (26,9\%), Bioquímica $(21,4 \%)$ e Farmácia $(19,7 \%)$. Quanto às atividades desenvolvidas pelos estudantes no momento da pesquisa, 60 (8,6\%) declararam estar trabalhando (além de estar estudando) e 403 (58,1\%) estavam vinculados a atividades extracurriculares. $O$ uso não prescrito de metilfenidato foi declarado por 30 participantes, correspondendo a 4,3\% da amostra do estudo.

A Tabela 1 apresenta as frequências absoluta e relativa das declarações desses quanto ao conhecimento sobre o metilfenidato e o perfil de uso praticado. A maioria dos estudantes declarou conhecer a indicação de uso do medicamento e a via de administração declarada foi somente a oral. Os principais motivos informados para o uso foram a necessidade de prestar mais atenção nos estudos e de melhorar o desempenho acadêmico. A universidade foi o principal local de início de uso e as principais motivações vinculadas ao ambiente acadêmico e escolar foram a melhora no desempenho e a necessidade de se concentrar nos estudos. O metilfenidato foi obtido principalmente por meio de amigos e não houve compras do medicamento em sites da internet.

No que diz respeito aos fatores associados com o uso não prescrito do metilfenidato, houve uma maior frequência em estudantes do sexo masculino $(9,2 \%)$, com idade maior que 21 anos (5,8\%), que cursavam Bioquímica $(7,4 \%)$ ou Medicina $(8,1 \%)$, que trabalhavam $(7,6 \%)$ e realizavam atividades extracurriculares $(6,7 \%)$. As variáveis sociodemográficas: religião, 
Tabela 1. Conhecimento do metilfenidato e perfil de uso não prescrito. São João Del-Rei, Minas Gerais-MG, Brasil, 2015-2016

\begin{tabular}{|c|c|c|}
\hline Variável & $\begin{array}{l}\text { Frequência } \\
\text { absoluta }\end{array}$ & $\begin{array}{l}\text { Frequência } \\
\text { relativa }(\%)\end{array}$ \\
\hline TOTAL & 30 & 100 \\
\hline \multicolumn{3}{|l|}{ Conhece a indicação do metilfenidato } \\
\hline Sim & 29 & 96,7 \\
\hline Não & 1 & 3,3 \\
\hline \multicolumn{3}{|l|}{ Momento da vida estudantil de início de uso do metilfenidato } \\
\hline Universidade & 18 & 60 \\
\hline Cursinho pré-vestibular & 9 & 30 \\
\hline Ensino médio & 1 & 3,3 \\
\hline Não respondeu & 2 & 6,7 \\
\hline \multicolumn{3}{|l|}{ Motivos de uso não médico do metilfenidato na vida' ${ }^{1}$} \\
\hline Ter mais atenção aos estudos & 23 & 76,6 \\
\hline Melhorar o desempenho acadêmico & 13 & 43,3 \\
\hline Ficar acordado & 11 & 36,6 \\
\hline Melhorar a memória & 8 & 26,6 \\
\hline Ter maior disposição no dia a dia & 6 & 20,0 \\
\hline Curiosidade & 6 & 20,0 \\
\hline Outros & 1 & 3,3 \\
\hline \multicolumn{3}{|l|}{ Motivos de uso na vida acadêmica/escolar do metilfenidato ${ }^{1}$} \\
\hline Quero melhorar meu desempenho acadêmico no geral & 14 & 46,6 \\
\hline Preciso tirar uma nota alta em certa prova/teste & 13 & 43,3 \\
\hline Não consigo me concentrar nos estudos & 12 & 40 \\
\hline Preciso ficar acordado para estudar & 7 & 23,3 \\
\hline Não tenho tempo para estudar devido a outras atividades & 4 & 13,3 \\
\hline Outros & 4 & 13,3 \\
\hline \multicolumn{3}{|l|}{ Meio de obtenção do metilfenidato ${ }^{1}$} \\
\hline Por meio de amigos & 14 & 46,6 \\
\hline Outros & 6 & 20 \\
\hline Parentes que necessitam do uso do medicamento & 5 & 16,6 \\
\hline Conheço um médico que me prescreve & 3 & 10 \\
\hline Em farmácia/drogaria que vende sem receita & 2 & 6,6 \\
\hline \multicolumn{3}{|l|}{ Motivo pelo qual interrompeu atualmente o uso do metilfenidato ${ }^{1,2}$} \\
\hline Não acho mais necessário & 12 & 40 \\
\hline Ocorreram reações indesejáveis (reações adversas) & 6 & 20 \\
\hline Medo de ficar dependente & 5 & 16,6 \\
\hline Ainda faço uso do metilfenidato & 3 & 10 \\
\hline $\begin{array}{l}\text { Não ocorreram reações adversas, mas fiquei com medo de que } \\
\text { ocorressem. }\end{array}$ & 3 & 10 \\
\hline Dificuldade financeira para comprar o medicamento & 2 & 6,6 \\
\hline Não atingi o efeito esperado & 2 & 6,6 \\
\hline Outros & 2 & 6,6 \\
\hline Dificuldade para obter o medicamento & 1 & 3,3 \\
\hline
\end{tabular}

1- Foi permitido assinalar mais de uma resposta entre as alternativas.

No momento do estudo, 27 dos 30 estudantes declararam não estar mais fazendo uso do metilfenidato

renda e trabalho não apresentaram significância estatística quanto ao uso não prescrito de metilfenidato (Valor de $\mathrm{P}>0,05$ ) (Tabela 2).

Na Tabela 3 estão apresentadas as variáveis que compuseram o modelo final da análise multivariada com o controle de confusão pelas demais. Houve uma maior chance de uso não prescrito de metilfenidato entre pessoas do sexo masculino, maiores de 21 anos e que 
Tabela 2. Distribuição da frequência de uso não prescrito de metilfenidato e características sociodemográficas. São João Del-Rei, Minas Gerais-MG, Brasil, 2015-2016

\begin{tabular}{|c|c|c|c|}
\hline & Uso (\%) & Não uso (\%) & Valor de $\mathbf{P}$ \\
\hline Total & 30 & 666 & - \\
\hline \multicolumn{4}{|l|}{ Sexo } \\
\hline Feminino & $12(2,4 \%)$ & $483(97,6 \%)$ & \multirow[t]{2}{*}{$<0,001$} \\
\hline Masculino & $18(9,2 \%)$ & $178(90,8 \%)$ & \\
\hline \multicolumn{4}{|l|}{ Idade } \\
\hline$<21$ anos & $5(1,9 \%)$ & $261(98,2 \%)$ & \multirow[t]{2}{*}{0,013} \\
\hline$>21$ anos & $25(5,8 \%)$ & $403(94,2 \%)$ & \\
\hline \multicolumn{4}{|l|}{ Religião } \\
\hline Segue & $24(4,1 \%)$ & $565(95,9 \%)$ & \multirow[t]{2}{*}{0,430} \\
\hline Não segue & $6(5,8 \%)$ & $97(94,2 \%)$ & \\
\hline \multicolumn{4}{|l|}{ Renda } \\
\hline$<R \$ 3.000,00$ & $8(3,0 \%)$ & $256(97,0 \%)$ & \multirow[t]{2}{*}{0,273} \\
\hline$>R \$ 3.000,00$ & $20(4,7 \%)$ & $20(4,7 \%)$ & \\
\hline \multicolumn{4}{|l|}{ Curso } \\
\hline Bioquímica & $11(7,4 \%)$ & $138(92,6 \%)$ & \multirow[t]{4}{*}{$<0,001$} \\
\hline Farmácia & $0(0,0 \%)$ & $137(100 \%)$ & \\
\hline Enfermagem & $1(0,5 \%)$ & $186(99,5 \%)$ & \\
\hline Medicina & $18(8,1 \%)$ & $204(91,9 \%)$ & \\
\hline \multicolumn{4}{|c|}{ Atualmente trabalhando } \\
\hline Sim & $5(7,6 \%)$ & $61(92,4 \%)$ & \multirow[t]{2}{*}{0,195} \\
\hline Não & $25(4,0 \%)$ & $599(96,0 \%)$ & \\
\hline \multicolumn{4}{|c|}{ Vinculado a atividades extracurriculares } \\
\hline Sim & $27(6,7 \%)$ & $376(93,3 \%)$ & \multirow[t]{2}{*}{0,000} \\
\hline Não & $3(1,0 \%)$ & $288(99,0 \%)$ & \\
\hline
\end{tabular}

Tabela 3. Fatores associados ao uso não prescrito de metilfenidato no modelo multivariado final. São João Del-Rei, Minas Gerais-MG, Brasil, 2015-2016

\begin{tabular}{|c|c|c|c|}
\hline Variável & OR & IC 95\% & Valor de P \\
\hline \multicolumn{4}{|l|}{ Sexo } \\
\hline Feminino & 1 & & 0,037 \\
\hline Masculino & 2,307 & $1,048-5,080$ & \\
\hline \multicolumn{4}{|l|}{ Idade } \\
\hline$<21$ anos & 1 & & 0,38 \\
\hline$\geq 21$ anos & 2,910 & 1,064-7,961 & \\
\hline \multicolumn{4}{|l|}{ Curso } \\
\hline Bioquímica & 1 & & \\
\hline Farmácia & 0 & & 0,995 \\
\hline Enfermagem & 0,68 & $0,009-0,549$ & 0,009 \\
\hline Medicina & 0,707 & $0,309-1,618$ & 0,412 \\
\hline \multicolumn{4}{|c|}{ Atividades extracurriculares } \\
\hline Não & 1 & & 0,006 \\
\hline Sim & 5,616 & $1,631-19,334$ & \\
\hline
\end{tabular}

OROdds Ratio; ICIntervalo de confiança

desempenham atividades extracurriculares. Em relação ao curso, identificou-se uma menor chance de uso em estudantes de Enfermagem e a maior chance em alunos de Bioquímica.

As Tabelas 4 e 5 expõem, respectivamente, os resultados obtidos quanto às percepções experimentadas pelos estudantes e os efeitos indesejados ocorridos por meio do uso não prescrito de metilfenidato. A maior parte dos estudantes universitários relatou ter percebido um aumento na concentração (80\%) e uma melhora do rendimento estudantil (50\%). 
Tabela 4. Percepções acerca do uso de metilfenidato. São João Del-Rei, Minas Gerais-MG, Brasil, 2015-2016

\begin{tabular}{lcc}
\multicolumn{1}{c}{ Variável } & $\begin{array}{c}\text { Frequência } \\
\text { absoluta }\end{array}$ & $\begin{array}{c}\text { Frequência } \\
\text { relativa (\%) }\end{array}$ \\
\hline TOTAL & $\mathbf{3 0}$ & 100 \\
\hline Aumento da concentração após uso de metilfenidato & 24 & \\
\hline Sim & 5 & 16,6 \\
\hline Não & & \\
\hline Melhora do rendimento estudantil após uso de metilfenidato & 15 & 50 \\
\hline Sim & 14 & 46,6 \\
\hline Não & & \\
\hline Maior capacidade de ficar acordado após uso de metilfenidato & 17 & 56,6 \\
\hline Não & 13 & 43,3 \\
\hline Sim & & 73,3 \\
\hline Melhora da memória após uso de metilfenidato & 22 & 26,6 \\
\hline Não & 8 & 80 \\
\hline Sim & & 20 \\
\hline Maior disposição no dia a dia após uso de metilfenidato & 24 & \\
\hline Não & 6 & \\
\hline Sim & & \\
\hline
\end{tabular}

Tabela 5. Efeitos indesejados após uso de metilfenidato. São João Del-Rei, Minas Gerais-MG, Brasil, 2015-2016

\begin{tabular}{lcc}
\hline \multicolumn{1}{c}{ Variável } & $\begin{array}{c}\text { Frequência } \\
\text { absoluta }\end{array}$ & $\begin{array}{c}\text { Frequência } \\
\text { relativa (\%) }\end{array}$ \\
\hline TOTAL & $\mathbf{3 0}$ & 100 \\
\hline Ocorrência de efeitos indesejados & & 50 \\
\hline Não & 15 & 43,3 \\
\hline Sim & 13 & 6,7 \\
\hline Não respondeu & 2 & 50 \\
\hline Efeitos indesejados percebidos após uso de Metilfenidato & & 33,3 \\
\hline Não tive efeitos indesejados & 15 & 13,3 \\
\hline Taquicardia & 10 & 13,3 \\
\hline Ansiedade & 4 & 10 \\
\hline Outros & 4 & 6,6 \\
\hline Insônia & 3 & 6,6 \\
\hline Perda de apetite & 2 & 3,3 \\
\hline Alterações gastrointestinais & 2 & 3,3 \\
\hline Tremores nas mãos & 1 & 3,3 \\
\hline Transpiração excessiva & 1 & 3,3 \\
\hline Alucinações & 1 & 3,3
\end{tabular}

Efeitos indesejados pelo uso não prescrito de metilfenidato foram declarados por $50 \%$ dos participantes, sendo o mais relatado a ocorrência de taquicardia (33,3\%).

\section{DISCUSSÃO}

Conforme observado na literatura científica, os debates acerca do uso não prescrito de metilfenidato têm se intensificado na última década². Tal discussão já é ampla internacionalmente e vem ganhando espaço no Brasil, principalmente devido à constatação de aumento no número de estudantes que recorrem a psicoestimulantes para melhorar o rendimento acadêmico, sendo esta questão um sério problema de saúde pública ${ }^{18}$. Além disso, a necessidade de realizar um grande número de tarefas em pouco tempo e com eficiência é 
considerada por universitários como um dos maiores motivos para as pessoas utilizarem o metilfenidato com fins'não médicos'18.

Encontrou-se uma prevalência de uso não médico do metilfenidato de $4,3 \%$ entre os universitários que compuseram a amostra deste estudo. Na revisão bibliográfica, os resultados das pesquisas nacionais apontam uma prevalência de $60 \%$ no estudo realizado no município de Campos dos Goytacazes/R ${ }^{22}$, de $27 \%$ no estudo no município de Volta Redonda/R ${ }^{23}$, de $0,9 \%$ em estudo realizado em 27 capitais brasileiras ${ }^{24}$, de $23 \%$ em estudo na região Sul do Brasi $^{25}$ e de 8,6\% em estudo na Bahia ${ }^{26}$. Uma hipótese para a heterogeneidade de resultados seriam as características específicas das amostras estudadas, bem como os diferentes contextos socioculturais nos quais os estudos foram conduzidos. Nas pesquisas internacionais, um estudo australiano $0^{27}$ encontrou prevalência de uso de 6,7\%; o estudo iraniano ${ }^{28}$ encontrou a prevalência de uso de $8,7 \%$; e o estudo suíço ${ }^{11}$ encontrou prevalência de uso de $5,8 \%$, resultados mais próximos dos encontrados nesta pesquisa.

Em relação aos fatores associados, no que diz respeito ao sexo, a maioria dos estudos nacionais, assim como este, demostram uma predominância de uso pelo sexo masculino. $O$ estudo suíço ${ }^{11}$ também encontrou uma maior prevalência de uso entre homens, embora as mulheres tenham tido um maior uso de neuroaprimorantes mais leves como fitoterápicos, vitaminas, café, cafeína e energéticos. A maior prevalência de uso não prescrito do metilfenidato em homens, parece sugerir que esses estariam mais sujeitos à pressão social para a melhoria de desempenho ${ }^{18}$, uma vez que este poderia estar mais associado aos atributos de masculinidade. A manutenção da variável sexo no modelo multivariado do presente estudo reforça o achado e indica que intervenções específicas para homens e mulheres podem ter resultados mais efetivos. Apesar da prevalência de uso ser maior nos homens, o grupo das mulheres merece atenção, pois as pesquisas indicam que elas são mais sensíveis aos efeitos psicoestimulantes e, em caso de dependência, apresentam maiores taxas de recaída e dificuldade para diminuir o consumo ${ }^{29}$.

No presente estudo não foi encontrada diferença estatisticamente significante comparando os períodos dos cursos analisados. No entanto, assim como em alguns dos estudos nacionais já mencionados ${ }^{22,24,25}$, a maior prevalência de uso se deu em maiores de 21 anos. Os maiores de 21 anos, geralmente estão matriculados em períodos mais adiantados do curso. Esses períodos estão quase sempre associados a uma maior densidade de matérias, atividades extracurriculares e estágios curriculares, o que pode justificar a maior prevalência de uso.

Um dos achados deste estudo foi a existência de uma forte associação entre chance de uso de metilfenidato e realização de atividades extracurriculares. As atividades extracurriculares são aquelas não obrigatórias no currículo dos cursos tais como participação em grupos de pesquisa, ligas acadêmicas, projetos de extensão e outras atividades oferecidas pela universidade. Como essas atividades se somam aos encargos obrigatórios, levantou-se a hipótese da sobrecarga de atividades e da disponibilidade de tempo como desencadeadores do uso de metilfenidato. No entanto, é necessário um estudo mais focado nesta questão para que se possa ter maior clareza da hipótese levantada.

O uso de metilfenidato sem prescrição em estudantes na área da saúde chama muita atenção na medida em que são formações que possuem amplo conhecimento sobre os medicamentos, sua ação e indicação.

É importante refletir, tanto no ambiente acadêmico quanto entre profissionais de saúde, que o uso não médico de metilfenidato com fins de melhoria de desempenho acadêmico ou profissional, tem relação com a atual lógica da medicalização e farmacologização. Essa lógica aponta que, na sociedade atual, as relações entre saúde e sociedade ganham contornos específicos e o conjunto de interesses em jogo nesses processos podem se mostrar, muitas vezes, contrários ao bem estar de pessoas e populações. O fenômeno da medicalização representa a ampliação da influência médica em diferentes esferas do cotidiano das pessoas pela oferta de diagnóstico e tratamento de problemas que anteriormente não eram da competência médica ${ }^{30-32}$. A medicalização associa-se ao processo de farmacologização que fortalece a crença de que todos os problemas têm origem biológica e podem ser tratados com a administração correta de medicamentos. A farmacologização faz a leitura de diferentes 
questões humanas em correspondência à necessidade de uma intervenção farmacológica, independentemente da existência de um diagnóstico médico ${ }^{30}$.

Embora alguns autores discutam que especificamente o fato de cursar Medicina seria um fator de risco para uso não prescrito do medicamento $22,23,25,26,28$, no presente estudo, com o resultado ajustado na análise multivariada, identificou-se que os cursos de Bioquímica e Medicina são os que apresentam maior força de associação ao uso não prescrito de metilfenidato. Dentre as variáveis analisadas na modelagem, somente a condução de atividades extracurriculares diferiu entre os cursos estudados. Assim, a condução dessas atividades, teve papel relevante no aumento da chance de uso do medicamento. $O$ resultado sugere que esse é um grupo a ser priorizado em atividades de promoção de saúde e qualidade de vida.

Outra informação trazida nos resultados desta pesquisa é o fato da universidade ser o principal local de início de uso, aspecto não investigado em nenhuma das pesquisas nacionais mencionadas neste estudo. Nesse sentido, destaca-se a necessidade de que a organização curricular nas universidades busque avaliar se as atividades oferecidas e as exigências acadêmicas impostas podem estar gerando sobrecarga aos estudantes.

Outro ponto que chama atenção no estudo são os efeitos indesejados pelo uso não prescrito de metilfenidato que foram declarados por $50 \%$ dos participantes. Os mais relatados foram ocorrência de taquicardia (33,3\%) e ansiedade (13\%). Esses efeitos colaterais necessitam de divulgação, pois podem colaborar para que haja mais precaução do uso indevido. No estudo realizado em Campos de Goytacazes/RJ houve uma prevalência de efeito colateral em 86,6\% dos participantes do estudo, sendo os efeitos mais relatados: insônia, irritabilidade, ansiedade e diminuição do apetite ${ }^{22}$. Em Volta Redonda/RJ foram percebidos efeitos semelhante por $65 \%$ dos participantes. Taquicardia, anorexia, xerostomia e ansiedade estão entre os efeitos mais elencados ${ }^{23}$. Dentre os estudos internacionais, no suíço, 27,1\% dos participantes relataram irritabilidade; $26,4 \%$, distúrbios do sono; $17,9 \%$, anorexia e $15,8 \%$, taquicardiaa ${ }^{11}$.

No estudo de 2014, feito com 150 universitários dos cursos de Farmácia e Medicina da faculdade de Campos dos Goytacazes-RJ'22, também identificou-se que a maioria dos universitários que usava o metilfenidato de forma não prescrita o obtinha de amigos. Nesse estudo, a maioria dos estudantes (86,6\%) percebeu efeitos colaterais, mas, mesmo assim, $57,7 \%$ afirmaram que, ao término do curso, pretendem continuar utilizando o metilfenidato ${ }^{23}$. Essa mesma tendência foi percebida entre os estudantes que participaram do presente estudo. Tais resultados parecem apontar também para o fato de que desconfortos ou problemas em relação ao uso do medicamento muitas vezes não são argumentos suficientes para interromper o uso, frente aos objetivos de melhoria de desempenho. Nesse sentido, a saúde parece ser menos importante do que o desempenho.

No presente estudo, o metilfenidato foi utilizado com objetivos principais de obter mais atenção nos estudos e melhorar o desempenho acadêmico, tendo sido percebido o efeito de aumento da concentração. Apesar de os estudos mostrarem que os universitários acreditavam alcançar melhora do desempenho acadêmico com o uso não prescrito de metilfenidato, um estudo experimental realizado em Nova York, EUA, concluiu que o aumento do nível de dopamina no SNC após uso de metilfenidato por pessoas saudáveis só ocorre caso haja interesse e motivação frente a um estímulo ${ }^{16}$. Ou seja, o aumento da atenção e melhoria do desempenho dependeriam do interesse pela tarefa, podendo ser esse o mecanismo responsável pelos efeitos percebidos do uso de metilfenidato por pessoas saudáveis ${ }^{31}$. Da mesma forma, um estudo universitário na Bélgica ${ }^{33}$ não encontrou diferença significativa de performance entre estudantes saudáveis que usaram metilfenidato e aqueles que receberam medicação placebo, mas encontrou melhor desempenho entre aqueles que acreditavam ter recebido metilfenidato em relação aos que acreditavam ter recebido placebo ${ }^{8,33}$. Tais estudos apontam para uma provável constatação de que o metilfenidato exerce efeito psicológico ou efeito placebo em indivíduos saudáveis ${ }^{8,31,33}$.

Os dados obtidos neste estudo corroboram a observação de que, nos últimos anos, tem havido uma tendência de uso não prescrito de metilfenidato entre universitários com vistas a melhorar o desempenho acadêmico dos. Os resultados mostraram que os universitários conhecem a substância e a sua indicação clínica e mais da metade deles informam efeitos 
colaterais após o uso não prescrito. Identifica-se a percepção de que existe uma melhora cognitiva associada ao consumo, o que não é confirmado pela literatura sobre o assunto. Notouse também que o início do consumo acontece na universidade e que os homens consomem mais que as mulheres.

O recurso ao metilfenidato para a melhoria do desempenho acadêmico deve ser amplamente discutido no ambiente universitário, pois ajuda a refletir que o acesso ao conhecimento científico pode não ser suficiente para desestimular o uso no campo que extrapole suas indicações formais de tratamento. Nesse sentido, torna-se possível promover ações e estratégias mais adequadas para evitar ou minimizar efeitos de abuso e adição consequentes do uso indiscriminado desse medicamento. Nesse contexto, os fatores associados ao uso, identificados no presente estudo, são relevantes para um melhor direcionamento das ações.

\section{REFERÊNCIAS}

1. Domitrovic N. As Práticas Farmacológicas com o Metilfenidato: habitando fronteiras entre o acesso e o excesso [dissertação]. Programa de Pós-Graduação em Psicologia Institucional, Universidade Federal do Espírito Santo; 2014 [acesso em 2016 Out 08]. Disponível em: https://repositorio.ufes.br/handle/10/2951

2. Shirakawa DM, Tejada SN, Marinho CAF. Questões atuais no uso indiscriminado do metilfenidato. Omnia Med. 2012;9(1):46-53.

3. Pastura G, Mattos P. Efeitos colaterais do metilfenidato. Rev Psiquiatr Clin (Santiago). 2004;31(2):100-4. http://dx.doi.org/10.1590/S0101-60832004000200006.

4. Smith ME, Farah MJ. Are prescription stimulants "smart pills"? The epidemiology and cognitive neuroscience of prescription stimulant use by normal healthy individuals. Psychol Bull. 2011;137(5):717-41. http://dx.doi. org/10.1037/a0023825. PMid:21859174.

5. Ortega F, Barros D, Caliman L, Itaborahy C, Junqueira L, Ferreira CP. A ritalina no Brasil: produções, discursos e práticas. Revista interface; 2010;14(34):499-510. http://dx.doi.org/10.1590/S1414-32832010005000003.

6. Sandra P, Maria FA, Ana A, Cláudia B, Virgínia M. Illicit use of Methylphenidate: the other side of the medical prescription. Nascer Crescer. 2018 jun;27(2):98-104.

7. Brasil. Agência Nacional de Vigilância Sanitária. Prescrição e consumo de metilfenidato no Brasil: identificando riscos para o monitoramento e controle sanitário. Boletim de Farmacologia do SNGPC. 2012;jul/dez;2(2):1-14. [acesso em 2017 Nov 21]. Disponível em https://crfms.org.br/upload/boletimde-farmacoepidemiologia.pdf

8. Beyer C, Staunton C, Moodley K. The implications of Methylphenidate use by healthy medical students and doctors in South Africa. BMC Med Ethics. 2014 mar;15(20):20. http://dx.doi.org/10.1186/1472-693915-20. PMid:24592964.

9. Teixeira M. Notícia preliminar sobre uma tendência contemporânea: o "aperfeiçoamento cognitivo", do ponto de vista da pesquisa em neurociências. Rev Latinoam Psicopatol Fundam. 2007;10(3):495-503. http://dx.doi.org/10.1590/1415-47142007003009.

10. Freese L, Signor L, Machado C, Ferigolo M, Barros HMT. Non-medical use of methylphenidate: a review. Trends Psychiatry Psychother. 2012;34(2):110-5. http://dx.doi.org/10.1590/S2237-60892012000200010. PMid:25922930.

11. Maier LJ, Liechti ME, Herzig F, Schaub MP. To dope or not to dope: neuroenhancement with prescription drugs and drugs of abuse among Swiss university students. PLoS One. 2013 nov;8(11):e77967. http:// dx.doi.org/10.1371/journal.pone.0077967. PMid:24236008.

12. Finger G, Silva E, Falavigna A. Use of methylphenidate among medical students: a systematic review. Rev Assoc Med Bras. 2013 maio/jun;59(3):285-9. http://dx.doi.org/10.1016/j.ramb.2012.10.007. PMid:23680277.

13. Internacional Narcotics Control Board. Psychotropic substances. Statistics for 2011. Assessments of Annual Medical and Scientific Requirements. USA: Internacional Narcotics Control Board; 2012 [acesso em 2014 Nov 8]. Disponível em: http://www.incb.org/documents/Psychotropics/technical-publications/2012/en/ Eng_2012_PUBlication.pdf

14. Habibzadeh A, Alizadeh M, Malek A, Maghbooli L, Shoja MM, Ghabili K. Illicit methiyphenidate use among Iranian medical students: prevalence and knowledge. Drug Des Devel Ther. 2011 fev;5:71-6. PMid:21340040. 
15. Repantis D, Schlattmann $P$, Laisney $O$, Heuser I. Modafinil and methylphenidate for neuroenhancement in healthy individuals: a systematic review. Pharmacol Res. 2010;62(3):187-206. http://dx.doi.org/10.1016/j. phrs.2010.04.002. PMid:20416377.

16. Volkow ND, Wang GJ, Fowler JS, Telang F, Maynard L, Logan J, et al. Evidence that methylphenidate enhances the saliency of a mathematical task by increasing dopamine in the human brain. Am J Psychiatry. 2004;161(7):1173-80. http://dx.doi.org/10.1176/appi.ajp.161.7.1173. PMid:15229048.

17. Rocha CEL, Wagner GA, Castaldelli-Maia JM, Silveira CM, Andrade AG, Oliveira LG. Uso prescrito de cloridrato de metilfenidato e correlatos entre estudantes universitários brasileiros. Rev Psiquiatr Clin (Santiago). 2012;39(6):183-8. https://doi.org/10.1590/S0101-60832012000600001.

18. Barros D, Ortega F. Metilfenidato e aprimoramento cognitivo farmacológico: representações sociais de universitários. Saude Soc. 2011 abr/jun;20(2):350-62. http://dx.doi.org/10.1590/S010412902011000200008.

19. Birman J. Que Droga! In: Birman, J. Mal estar na atualidade. São Paulo: Civilização Brasileira; 2000.

20. Brant LC, Carvalho TRF. Metilfenidato: medicamento gadjet da contemporaneidade. Interface - Comunic, Saúde Educ. 2012;16(42):623-36. https://doi.org/10.1590/S1414-32832012000300004.

21. Dantas JB. Tecnificação da vida: uma discussão sobre o discurso da medicalização da sociedade. Fractal. Rev Psicol. 2009;21(3):563-80. https://doi.org/10.1590/S1984-02922009000300011.

22. Pessanha FF, Mota JS. Prevalência do uso de Metilfenidato por universitários de Campos dos Goytacazes, RJ. Vértices. 2014;16(1):77-86. http://dx.doi.org/10.5935/1809-2667.20140005.

23. Carneiro SG, Prado AST, Araujo ECJ, Moura HC, Strapasson JF, Rabelo NF, et al. O uso não prescrito de metilfenidato entre acadêmicos de Medicina. Cadernos UniFOA. 2013;8(1 N. esp.):53-9. https://doi. org/10.47385/cadunifoa.v8.n1\%20(Esp.).87

24. Rocha CELD, Arantes WG, Maurício C-MJ, Magalhães SC, Andrade AGD, Oliveira LGD. Uso prescrito de cloridrato de metilfenidato e correlatos entre estudantes universitários brasileiros. Rev Psiquiatr Clin (Santiago). 2012;39(6):183-8.

25. Silveira RR, Lejderman B, Ferreira PEMS, Rocha GMP. Patterns of non-medical use of methylphenidate among 5th and 6th year students in a medical school in southern Brazil. Trends Psychiatry Psychother. 2014 jun;36(2):101-6. http://dx.doi.org/10.1590/2237-6089-2013-0065. PMid:27000709.

26. Cruz TCSC, Barreto EPS Jr, Gama MLM, Maia LCM, Melo MJX Fo, Manganotti O No, Coutinho DM. Uso não prescrito de metilfenidato entre estudantes de Medicina da Universidade Federal da Bahia. Gaz Méd Bahia. [Internet]. 2011;81(1):3-6. [acesso em 2017 Jan 13]. Disponível em: http://www.gmbahia.ufba.br/ index.php/gmbahia/article/viewFile/1148/1082

27. Mazanov J, Dunn M, Connor J, Fielding M. Substance use to enhance academic performance among Australian university students. Perform Enhanc Health. 2010;2(3):110-8. http://dx.doi.org/10.1016/j. peh.2013.08.017.

28. Habibzadeh A, Alizadeh M, Malek A, Maghbooli L, Shoja MM, Ghabili K. Illicit methylphenidate use among Iranian medical students: prevalence and knowledge. Drug Des Devel Ther. 2011;5:71-6. PMid:21340040.

29. Becker JB, Chartoff E. Sex differences in neural mechanisms mediating reward and addiction. Neuropsychopharmacology. 2019;44(1):166-83. http://dx.doi.org/10.1038/s41386-018-0125-6. PMid:29946108.

30. Camargo KR Jr. Medicalização, farmacologização e imperialism sanitário. Cad Saude Publica. 2013 maio;29(5):844-6. http://dx.doi.org/10.1590/S0102-311X2013000500002. PMid:23702990.

31. Monteiro B, Oliveira K, Rodrigues L, Fernandes T, Silva J, Viana N, et al. Metilfenidato e melhoramento cognitivo em universitários. SMAD Rev Eletrônica Saúde Mental Álcool Drog. 2018;13(4):232-42. http:// dx.doi.org/10.11606/issn.1806-6976.v13i4p232-242.

32. Tesser CD, Poli No P, Campos GWS. Acolhimento e (des)medicalização social: um desafio para as equipes de saúde da família. Ciênc Saúde Coletiva. 2010 nov;15(Supl 3):3615-24. https://doi.org/10.1590/S141381232010000900036 .

33. Mommaerts J-L, Beerens G, Van den Block L, Soetens E, Schol S, Van De Vijver E, et al. Influence of methylphenidate treatment assumptions on cognitive function in healthy young adults in a double-blind, placebo-controlled trial. Psychol Res Behav Manag. 2013 ago;6:65-74. http://dx.doi.org/10.2147/PRBM. S47526. PMid:24039459. 